Pesq. Vet. Bras. 37(12):1373-1379, dezembro 2017 DOI: $10.1590 / \mathrm{S} 0100-736 \mathrm{X} 2017001200003$

\title{
Identificação por espectrometria de massa MALDI-TOF de Salmonella spp. e Escherichia coli isolados de carcaças bovinas $^{1}$
}

\author{
Daniele Bier ${ }^{2,3}$, Juliane F. Tutija ${ }^{3}$, Taynara N. Pasquatti ${ }^{3}$, Tayná L. Oliveira ${ }^{3}$, Flábio R. \\ Araújo ${ }^{4}$ e Newton V. Verbisck ${ }^{4 *}$
}

\begin{abstract}
Bier D., Tutija J.F., Pasquatti T.N., Oliveira T.L., Araújo F.R. \& Verbisck N.V. 2017. [MALDI-TOF mass spectrometry identification of Salmonella spp. and Escherichia coli isolated from bovine carcasses.] Identificação por espectrometria de massa MALDI-TOF de Salmonella spp. e Escherichia coli isolados de carcaças bovinas. Pesquisa Veterinária Brasileira 37(12):1373-1379. Setor de Sanidade Animal, Embrapa Gado de Corte, Av. Rádio Maia 830, Zona Rural, Campo Grande, MS 79106-550, Brazil. E-mail: newton.verbisck@embrapa.br

The aim of this study was to introduce matrix-assisted laser desorption/ionization (MALDI) time-of-flight (TOF) mass spectrometry to improve the traditional microbiological method for the detection of Salmonella spp. and Escherichia coli in beef carcasses. Two hundred seventy samples from 90 beef carcasses were evaluated. The methodologies described in ISO 6579:2002 and in the Compendium of Methods for the Microbiological Examination of Foods were used for Salmonella spp. and E. coli isolation, respectively. MALDI-TOF analysis were performed on tryptone soya broth suspension isolates or directly from nutrient agar colonies, from the positive, inconclusive or negative biochemically tested samples for Salmonella and E. coli. Mass profiles were acquired on an Autoflex III SmartBeam MALDI-TOF mass spectrometer and the raw spectra were processed using the MALDI Biotyper software (Bruker Daltonics). According to the preliminary identification based on colony morphology and the biochemical reactions, seven isolates were positive for Salmonella spp. Through MALDI Biotyper these seven isolates were also classified as belonging to the genus Salmonella and further identified as $S$. enterica. Four isolates showing unusual phenotypic characteristics and inconclusive results in biochemical tests for Salmonella were identified as belonging to Citrobacter and Proteus genera after MALDI analysis. Regarding Escherichia coli, 37 were positive for species biochemical testing which MALDI Biotyper confirmed. MALDI-TOF methodology allowed rapid Salmonella spp. and E. coli identity confirmation and may be used to detect these microrganisms within bacterial isolates from beef carcasses.
\end{abstract}

INDEX TERMS: MALDI-TOF, mass spectrometry, Salmonella spp., Escherichia coli, bovine carcasses, slaughterhouse, enterobacteria.

RESUMO.- 0 objetivo deste trabalho foi introduzir a técnica de espectrometria de massa com fonte de ionização e dessorção a laser assistida por matriz e analisador de

\footnotetext{
${ }^{1}$ Recebido em 21 de outubro de 2016.

Aceito para publicação em 1 de abril de 2017

${ }^{2}$ Faculdade de Medicina Veterinária e Zootecnia (FAMEZ), Universidade Federal de Mato Grosso do Sul (UFMS), Av. Senador Felinto Müller 2443, Pioneiros, Campo Grande, MS 79070-900, Brasil.

${ }^{3}$ Curso de Medicina Veterinária, Universidade Católica Dom Bosco (UCDB), Av. Tamandaré 6000, Jardim Seminário, Campo Grande, MS 79117-900, Brasil.

${ }^{4}$ Setor de Sanidade Animal, Embrapa Gado de Corte, Av. Rádio Maia 830, Zona Rural, Campo Grande, MS 79106-550, Brasil. *Autor para correspondência: newton.verbisck@embrapa.br
}

tempo-de-voo (MALDI-TOF) para incrementar o método tradicional microbiológico na detecção de Salmonella spp. e Escherichia coli em carcaças bovinas. Foram avaliadas 270 amostras de 90 carcaças de bovinos. Para isolamento de Salmonella spp. e E. coli, foram utilizadas, respectivamente, as metodologias descritas na ISO 6579:2002 e no Compendium of Methods for the Microbiological Examination of Foods. As análises por MALDI-TOF foram realizadas a partir de isolados cultivados em ágar nutriente ou em caldo triptona de soja, provenientes das amostras com características bioquímicas positivas $(\mathrm{n}=7)$, inconclusivas $(\mathrm{n}=4)$ e negativas $(\mathrm{n}=85)$ para Salmonella spp. e bioquímicas positivas $(\mathrm{n}=37)$ e negativas $(\mathrm{n}=85)$ para $E$. coli. Os 
perfis de massas foram adquiridos com o espectrômetro de massas MALDI-TOF Autoflex III SmartBeam e os espectros brutos foram processados usando o programa MALDI Biotyper (Bruker Daltonics). De acordo com a identificação preliminar, com base na morfologia das colônias e nas reações bioquímicas, sete isolados foram considerados positivos para Salmonella spp. Através do MALDI Biotyper, esses sete isolados foram classificados como pertencentes ao gênero Salmonella e, além disso, identificados como S. enterica. Quatro isolados que apresentaram características fenotípicas não usuais e resultados inconclusivos nos testes bioquímicos para Salmonella foram identificados como pertencentes aos gêneros Citrobacter e Proteus após análise por MALDI. Para E. coli, 37 amostras foram positivas pelos testes bioquímicos da espécie, o que foi confirmado por MALDI Biotyper. A metodologia MALDI-TOF permitiu a rápida confirmação da identidade de Salmonella spp. e E. coli, podendo ser utilizada para detecção desses microrganismos em isolados bacterianos de carcaças bovinas.

TERMOS DE INDEXAÇÃO: Espectrometria de massa, MALDI-TOF, Salmonella spp., Escherichia coli, carcaça bovina, bovinos, abatedouro-frigorífico, enterobactérias.

\section{INTRODUÇÃO}

A carne é um dos alimentos mais importantes da dieta da população e possui importância fundamental para a economia do Brasil, que é um grande produtor mundial de proteína animal e o maior exportador de carne bovina do mundo (Brasil 2016). Porém, a carne e seus derivados são considerados um dos principais responsáveis pela veiculação de patógenos ao homem (Rhoades et al. 2009, Filippis et al. 2013), ocasionando as chamadas doenças transmitidas por alimentos.

Durante o abate, os animais de corte podem ter suas carcaças contaminadas por bactérias, veiculando esses microrganismos nos cortes das carnes ou nos produtos processados. A contaminação pode ocorrer a partir de bactérias presentes na carcaça externa, a partir do trato intestinal ou linfonodos do animal abatido, seja durante as etapas de abate, transporte, armazenamento e distribuição da carne, ou pela manipulação humana sem condições higiênicas (Borch \& Arinder 2002, Catellani et al. 2014). Enquanto a maioria das bactérias é inofensiva e causa apenas deterioração do alimento, bactérias patogênicas como Escherichia coli e Salmonella enterica podem também estar presentes (Rhoades et al. 2009, Filippis et al. 2013, EFSA 2015).

Os métodos convencionais de detecção dessas bactérias em alimentos ainda são considerados como oficiais em diversos países (APHA 1992, Meng et al. 2001, ISO 2002, FDA 2014) e no Brasil (Brasil 2003). Essas técnicas tradicionais de microbiologia envolvem etapas de cultura onerosas e bastante trabalhosas, necessitando de até sete dias para a confirmação dos resultados, pois são necessárias as etapas de pré-enriquecimento, enriquecimento seletivo, plaqueamento seletivo diferencial e confirmação bioquímica e sorológica. Essas etapas servem para aumentar a recuperação das células nos alimentos que possuem microbiota competitiva, células em número reduzido ou injuriadas (Havelaar et al. 2010, Lopez-Velasco et al. 2015). Além disso, a identificação por perfis fenotípicos utilizada nessas metodologias é propensa a erros devido à possibilidade de variabilidade desses perfis, levando a reações falso-negativas, ou ainda ser de difícil ou errônea interpretação (Marin et al. 2006, Settanni \& Corsetti 2007, Carrique-Mas \& Davies 2008, Spanu et al. 2011).

Diversos métodos rápidos para detecção de Salmonella e E. coli são comercialmente disponíveis e podem ser classificados como procedimentos convencionais adaptados ou modificados, ensaios baseados em imunologia e ensaios baseados em ácidos nucleicos. Destes métodos, o ensaio de imunoabsorção enzimática (ELISA) e a Reação em Cadeia da Polimerase (PCR) mostram especificidade e sensibilidade comparável aos métodos convencionais, porém dependem em grande parte da microflora e matriz da amostra, da presença de células não cultiváveis e de substâncias inibidoras, tais como gorduras, proteínas, polissacarideos, metais pesados, antibióticos e compostos orgânicos (Lee et al. 2015).

Um grande avanço na detecção rápida de patógenos em alimentos pode ser o uso de estudos proteômicos, empregando-se a espectrometria de massa, para caracterização dos microrganismos alvos. A técnica de espectrometria de massa com fonte de ionização e dessorção a laser assistida por matriz - MALDI (Matrix Assisted Laser Desorption/ Ionization) e analisador de massas do tipo tempo-de-voo - TOF (Time-of-Flight), permite a comparação do espectro de massas de um microrganismo isolado com os espectros de referência de cepas conhecidas, possibilitando a classificação e identificação do patógeno com mais rapidez do que os métodos convencionais (Cherkaoui et al. 2010, Angeletti 2016).

Assim, o objetivo deste trabalho foi introduzir a técnica de espectrometria de massa MALDI-TOF para incrementar o método tradicional microbiológico na detecção de Salmonella spp. e E. coli em carcaças bovinas de abatedouros-frigoríficos exportadores de Mato Grosso do Sul.

\section{MATERIAL E MÉTODOS}

Amostras. Para a coleta das amostras foram utilizadas esponjas esterilizadas (Speci-Sponge ${ }^{\circledR}$ - Nasco, EUA) hidratadas com 10mL de Água Peptonada Tamponada 1\% (APT 1\%), sendo em cada esponja coletada uma amostra. Para a coleta, as esponjas foram friccionadas nas superfícies das carcaças (método não destrutivo), nas regiões do peito, coxão e vazio de cada carcaça, e acondicionadas em bolsas plásticas estéreis. Foram coletadas 270 amostras de 90 carcaças de bovinos, em três abatedouros-frigoríficos, localizados no Estado de Mato Grosso do Sul, que funcionam sob Inspeção Federal.

Cepas da Coleção de Microrganismos de Referência em Vigilância Sanitária (CMRVS) (INCQS-FIOCRUZ, Rio de Janeiro, RJ), foram utilizadas como controles negativos e positivos: Escherichia coli INCQS 00033 (ATCC 25922 - Biotipo I, genérica), Escherichia coli INCQS 00171 (CDC EDL-933, Verotoxigênica), Escherichia coli NEWPROV $^{\circledR}$ (ATCC 25922), Salmonella enterica subsp. enterica sorovar Enteritidis INCQS 00258 (ATCC 13076) e Salmonella enterica subsp. enterica sorovar Typhimurium NEWPROV ${ }^{\circledR}$ (ATCC 14028). 
Isolamento bacteriano. A cada bolsa plástica contendo a esponja foram adicionados $200 \mathrm{~mL}$ de APT 1\%. A mistura foi homogeneizada com auxílio de um homogeneizador de amostras (tipo Stomacher) por 60 segundos e colocada em frascos de Erlenmeyer, sendo esta a amostra inicial. Esses frascos foram incubados a $37 \pm 1^{\circ} \mathrm{C}$ por $18 \pm 2 \mathrm{~h}$.

Para detecção de Salmonella spp. foi utilizada a metodologia descrita no International Organization for Standardization (ISO 2002), com modificações, adicionando as provas bioquímicas de motilidade, produção de $\mathrm{H}_{2} \mathrm{~S}$, vermelho de metila e citrato.

A metodologia utilizada para a detecção de E. coli, foi a descrita no Compendium of Methods for the Microbiological Examination of Foods, 2001 (Meng et al. 2001). As colônias suspeitas de E. coli em ágar MacConkey Sorbitol (SMAC) foram isoladas em ágar nutriente, incubadas a $35 \pm 1^{\circ} \mathrm{C}$ por 18 a $24 \mathrm{~h}$ e submetidas às provas bioquímicas complementares de indol, ureia, motilidade, produção de $\mathrm{H}_{2} \mathrm{~S}$, vermelho de metila, Voges-Proskauer, fermentação de carboidratos (Triple Sugar Iron) e citrato.

Espectrometria de massa MALDI-TOF. Os perfis proteicos foram obtidos em triplicata, a partir das culturas bacterianas, de acordo com o protocolo de extração com etanol/ácido fórmico, descrito por Sauer et al. (2008) e revisto por Freiwald \& Sauer (2009). Foram coletados duzentos microlitros do caldo de cultura, que foram lavados duas vezes com água Milli-Q (Merck Millipore) estéril, por centrifugação a $16000 \mathrm{~g}$ durante $1 \mathrm{~min}$. Após descarte do sobrenadante, o precipitado celular foi cuidadosamente ressuspenso em $300 \mu \mathrm{L}$ de água Milli-Q estéril. Em seguida, as bactérias foram inativadas com a adição de $900 \mu \mathrm{L}$ de etanol absoluto (Sigma-Aldrich). Após centrifugação a $16000 \mathrm{~g}$ durante $2 \mathrm{~min}$, o sobrenadante foi desprezado e o sedimento foi seco ao ar à temperatura ambiente durante $10 \mathrm{~min}$. As proteínas celulares foram extraídas adicionando-se ao sedimento o volume de $10 \mu \mathrm{L}$ de ácido fórmico a 70\% (Sigma-Aldrich) e, após homogeneização, adição de $10 \mu \mathrm{L}$ de acetonitrila pura (Sigma-Aldrich). Após nova centrifugação a $16000 \mathrm{~g}$ durante $2 \mathrm{~min}$ aplicou-se $1 \mu \mathrm{L}$ do sobrenadante em poço da placa de MALDI (Bruker Daltonics), deixando-se secar ao ar ambiente. Cada amostra foi coberta com $1 \mu \mathrm{L}$ da matriz para MALDI, o ácido $\alpha$-ciano-4-hidroxicinâmico (Sigma-Aldrich), na concentração de $5 \mathrm{mg} / \mathrm{mL}$ em solução contendo $50 \%$ de acetonitrila e 2,5\% de ácido trifluoracético (v/v), e subsequente secagem ao ar.

Os espectros de massas foram adquiridos cm um espectrômetro de massas Autoflex III SmartBeam (Bruker Daltonics) equipado com um laser de $200 \mathrm{~Hz}$, em modo linear positivo, faixa de massa de 2.000 a 20.000 Daltons e calibração externa utilizando-se a mistura de calibrantes padrão Bacterial Test Standard (Bruker Daltonics). Os parâmetros do aparelho utilizados foram: tensão da fonte IS1 20kV, tensão da fonte IS2 de 18,55kV, tensão de lentes $8,80 \mathrm{kV}$ e tempo de retardo de extração de íons de $240 \mathrm{~ns}$. Foram realizados disparos de laser aleatórios com taxa de amostragem de picos de 0,5GS/s, totalizando-se 2000 espectros, que foram somados e processados pelo algoritmo de detecção de pico tipo centroide do programa FlexControl 3.3 (Bruker Daltonics), gerando o espectro bruto de cada amostra.

Identificação por MALDI Biotyper. Os espectros brutos foram processados usando o programa MALDI Biotyper 3.1 (Bruker Daltonics) com as configurações padrão. 0 programa realiza normalização, suavização, subtração da linha de base e colheita de picos, criando uma lista dos picos mais significativos do espectro bruto (valores de $\mathrm{m} / \mathrm{z}$ e respectiva intensidade, com a relação sinal/ruído maior do que 3, limiar mínimo de $0,1 \%$ do pico mais alto e um máximo de 100 picos).

Para identificar as bactérias desconhecidas, cada lista de picos gerada foi comparada diretamente com a lista de picos dos espectros de referência, denominados MSP (Main Spectra Projections), presentes na biblioteca IVD (In Vitro Diagnostic System, Bruker Daltonics) integrada, por meio do algoritmo Biotyper. Nesse processamento, realiza-se a correspondência de padrões de picos, utilizando a posição, as distribuições de intensidade e a frequência de picos, atribuindo-se os escores de classificação sem qualquer intervenção do utilizador. As análises por Biotyper são classificadas utilizando os valores de escore propostos pelo fabricante: uma pontuação entre 2,300 e 3,000 indica a identificação confiável de espécie; uma pontuação entre 2,000 e 2,299 indica a identificação confiável de gênero e provável identificação de espécie, uma pontuação entre 1,700 e 1,999 indica provável identificação de gênero e uma pontuação abaixo de 1,700 indica que não há identificação confiável.

À biblioteca IVD, contendo espectros de referência para 3.476 espécies, incluindo 16 cepas de Salmonella e 11 de E. coli, foram incluídos os MSP das cinco cepas controle da CMRVS citadas anteriormente. Cada MSP foi gerado a partir do processamento dos espectros de 32 replicatas, resultando em um espectro consenso dos picos mais reprodutíveis, que reflete o padrão proteico típico daquela cepa bacteriana. Para criar as listas de picos dos MSP das cepas controle, o programa Biotyper foi usado conforme descrito acima, utilizando-se 70 picos independentes com frequência mínima de $50 \%$.

Os dendrogramas foram construídos com o programa estatístico Matlab 7.1 (The MathWorks Inc.), integrado ao programa MALDI Biotyper 3.1, com os parâmetros: medida de distância por correlação e ligação por média, sendo esta normalizada entre a distância 0 (concordância total) e 1000 (sem concordância).

\section{RESULTADOS}

Ao confrontar os MSP produzidos para as cepas da CMRVS com a biblioteca IVD, foi possível identificar corretamente todas as cinco cepas controle testadas neste estudo, constatando a confiabilidade da técnica MALDI Biotyper em identificar Salmonella e Escherichia coli de modo preciso. Além disso, foram escolhidas aleatoriamente, a partir das 270 amostras, 85 isolados com perfis bioquímicos não compatíveis com Salmonella spp. ou E. coli. Esses 85 isolados foram identificados como outros gêneros bacterianos (Klebsiella, Pseudomonas, Enterobacter, Morganella, Citrobacter, Proteus e Providencia) após análise por MALDI Biotyper.

Com base na morfologia da colônia e nas reações bioquímicas, das 270 amostras coletadas de 90 carcaças em três abatedouros-frigoríficos, foram encontrados 7 isolados positivos para Salmonella. Buscando a confirmação do gênero e a eventual identificação de espécie para Salmonella, esses sete isolados bioquimicamente positivos para Salmonella spp., foram analisados por MALDI Biotyper. Nessa análise foi possível discriminar entre 3476 isolados de bactérias, incluindo-se 16 sorovares de Salmonella, além das duas cepas $S$. Enteritidis e $S$. Typhimurium da CMRVS, cultivados e isolados em paralelo neste trabalho como controle. A técnica de MALDI Biotyper confirmou a identificação do gênero Salmonella para todos os 7 isolados analisados, classificando-os como sendo da espécie $S$. enterica (Quadro 1). Além disso, esses resultados revelaram que 6 dos 7 isolados apresentaram maior escore de identificação com o sorovar $S$. Enteritidis, enquanto que apenas o isolado 67 apresentou maior similaridade com a cepa controle correspondente ao sorovar $S$. Choleraesuis. Utilizando-se 
Quadro 1. Identificação por MALDI Biotyper dos isolados de carcaças bovinas positivos bioquimicamente para Salmonella spp.

\begin{tabular}{|c|c|c|c|c|c|c|}
\hline Isolado & Réplica 1 & Escore & Réplica 2 & Escore & Réplica 3 & Escore \\
\hline 7 & Salmonella Enteritidis & 2,414 & Salmonella Enteritidis & 2,396 & Salmonella Enteritidis & 2,368 \\
\hline 11 & Salmonella Enteritidis & 2,445 & Salmonella sp (choleraesuis)* & 2,363 & Salmonella Enteritidis & 2,348 \\
\hline 33 & Salmonella Enteritidis & 2,42 & Salmonella Enteritidis & 2,401 & Salmonella Enteritidis & 2,396 \\
\hline 38 & Salmonella Enteritidis & 2,477 & Salmonella Enteritidis & 2,449 & Salmonella Enteritidis & 2,365 \\
\hline 45 & Salmonella sp (choleraesuis)* & 2,352 & Salmonella Enteritidis & 2,335 & Salmonella Enteritidis & 2,304 \\
\hline 53 & Salmonella Enteritidis & 2,448 & Salmonella Enteritidis & 2,376 & Salmonella Enteritidis & 2,319 \\
\hline 67 & Salmonella sp (choleraesuis)* & 2,316 & Salmonella sp (choleraesuis)* & 2,314 & Salmonella Enteritidis & 2,264 \\
\hline
\end{tabular}

* Nomenclatura presente na biblioteca utilizada no programa Biotyper.

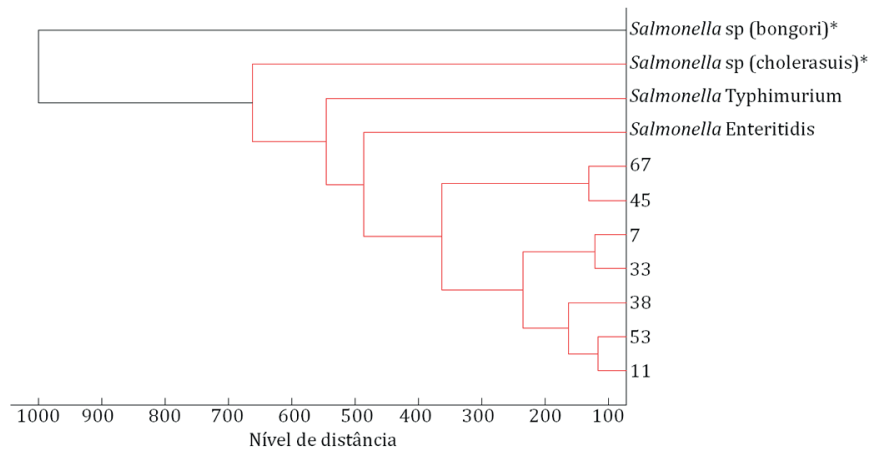

Fig.1. Dendrograma de isolados positivos para Salmonella spp. após análise por MALDI Biotyper.

as ferramentas integradas do pacote computacional MALDI Biotyper 3.1, construiu-se um dendrograma para ilustrar a semelhança molecular dos isolados frente às cepas de referência testadas, que indicou uma estreita proximidade entre os isolados e a cepa de referência de Salmonella Enteritidis (Fig.1).

Também foram encontrados 4 isolados com reações duvidosas nos testes bioquímicos que, por apresentarem características fenotípicas não usuais, foram consideradas de identificação inconclusivas para Salmonella. Esses quatro isolados foram analisados por MALDI Biotyper, tendo sido identificados como pertencentes aos gêneros Citrobacter e Proteus (Quadro 2). Na Figura 2 é possível verificar as diferenças de massa e intensidade dos picos que claramente distinguem os perfis proteicos dos gêneros Salmonella, Citrobacter e Proteus. No entanto, os escores dos isolados identificados como Citrobacter e Proteus ficaram abaixo de 2,300 , o que não permitiu identificar a espécie, provavelmente pela ausência na biblioteca das cepas de referência correspondentes a esses isolados. É possível ainda que esses isolados não tenham sido cultivados nas condições específicas, uma vez que o cultivo foi direcionado para o isolamento do gênero Salmonella.

Em relação à análise para $E$. coli, das 270 amostras coletadas provenientes das 90 carcaças, 37 foram positivas pelos testes bioquímicos para essa espécie. A análise por MALDI Biotyper mostrou que, conforme esperado, todos os isolados foram classificados no gênero Escherichia, enquanto que, para a identificação de espécie, 30 dos 37 isolados foram confirmados, ou seja, $81,1 \%$ dos casos (Quadro 3). Sete isolados (19,9\%) de Escherichia não tiveram a confirmação da espécie na análise por MALDI Biotyper, em razão de apresentarem escores entre 2,000 e 2,299, o que sugere que es- ses isolados correspondam a espécies ou sorovares não presentes na biblioteca IVD utilizada nestas análises.

\section{DISCUSSÃO}

A espectrometria de massa MALDI-TOF tem sido utilizada com sucesso para a identificação de uma grande variedade de espécies bacterianas (Clark et al. 2013). 0 espectro de massas gerado fornece um perfil proteômico do microrganismo desconhecido, tal como uma "impressão digital". Esses perfis são únicos para cada espécie de microrganismo, sendo possível inclusive distinguir picos específicos para os gêneros e espécies (Angeletti 2016). No caso da análise por MALDI Biotyper, cada perfil pode ser automaticamente comparado a uma biblioteca de espectros de referência, gerando a lista dos microrganismos mais estreitamente relacionados. Esse ranking indica o nível de confiança na identificação e, dependendo de quão elevado é o valor, o organismo é identificado no nível de gênero ou espécie (Patel 2013). Quando os espectros adquiridos para uma determinada amostra ou isolado não são identificados na biblioteca disponível, é possível gerar os correspondentes espectros de referência e incluí-los ao banco de dados, sendo o operador responsável pelo fornecimento ao sistema das informações precisas quanto à origem e referência daquele microrganismo. Neste estudo foi possível identificar com precisão todos os controles incluídos na biblioteca Biotyper, constatando a aptidão da técnica de MALDI-TOF em identificar corretamente Salmonella e E. coli.

Todos os isolados bioquimicamente positivos para Salmonella spp. foram identificados por MALDI Biotyper como pertencentes ao gênero Salmonella, enquanto que os isolados com perfis bioquímicos não compatíveis com Salmonella spp. foram identificados como outros gêneros bacterianos. De acordo com os valores de escore obtidos, as amostras de Salmonella foram $100 \%$ correlacionadas ao nível de gênero e identificadas como sendo da espécie $S$. enterica. Diversas investigações também relatam identificação de aproximadamente $100 \%$ de alguns microrganismos, como o trabalho de Faron et al. 2015. Esses autores, utilizando a técnica MALDI Biotyper, avaliaram a capacidade de identificação de 2263 isolados bacterianos aeróbios Gram-negativos, sendo 23 gêneros e 61 espécies, encontrando a identificação correta de 99,8\% para gênero e 98,2\% para o nível de espécie. Além desse relato, também Spanu et al. 2011, empregaram MALDI Biotyper para avaliar 450 espécies estafilococócicas, conseguindo demonstrar a identificação correta de 447 espécies $(99,3 \%)$, além de todas as subespécies estudadas. 
A análise por MALDI Biotyper deste estudo possibilitou identificar todos os isolados de Salmonella como S. enterica. Além disso, seis dos sete isolados apresentaram maior escore de identificação para a cepa de referência $S$. Enteritidis, enquanto que apenas um isolado apresentou maior similaridade com a cepa de referência $S$. Choleraesuis. Porém, os resultados obtidos não permitem a classificação inequívoca dos sorovares desses isolados, o que talvez possa ser alcançado com maior quantidade de informação estrutural, por exemplo, através do sequenciamento de peptídeos dos perfis proteicos encontrados, conforme descrito por Fagerquist et al. 2014 e Hart et al. 2015.

A identificação bioquímica, mesmo após as etapas do cultivo tradicional de pré-enriquecimento, enriquecimento seletivo e plaqueamento diferencial, foi inconclusiva para quatro isolados. Os testes bioquímicos identificam as salmonelas por seus perfis fenotípicos, que podem eventualmente não ser expressos ou apresentar variabilidade devido à ação de fatores ambientais sobre a expressão gênica, implicando em resultados falso-negativos, ou de difícil interpretação (Marin et al. 2006, Settanni \& Corsetti 2007, Spanu et al. 2011). De fato, quando submetidos a testes mo- leculares de reação em cadeia da polimerase, os resultados desses quatro isolados indicaram não se tratar do gênero Salmonella (dados não mostrados), enquanto que a análise por MALDI Biotyper identificou-os como pertencentes aos gêneros bacterianos Citrobacter e Proteus.

Vários estudos já foram realizados para avaliar o desempenho de MALDI-TOF MS na identificação de microrganismos, demonstrando que essa metodologia apresenta uma alta acurácia para uma grande variedade de espécies (Eigner et al. 2009, Angeletti 2016) e que os bancos de dados comercialmente disponíveis Biotyper (Bruker Daltonics) e SARAMIS (Shimadzu Corporation) são confiáveis (Cherkaoui et al. 2010). Esses trabalhos comparam a espectrometria de massa com outras ferramentas já utilizadas na identificação de microrganismos, como os testes bioquímicos automatizados e os tradicionais de cultivo, e demonstram acurácias entre 98 e $99 \%$ para as espécies analisadas. Nagy et al. (2009), trabalhando com espécies de Bacteroides, relataram que o poder de discriminação e identificação por MALDI-TOF foi superior aos testes bioquímicos. Eigner et al. (2009), ao analisarem 1116 isolados clínicos comparando MALDI Biotyper com a identificação bioquímica conven-

Quadro 2. Identificação por MALDI Biotyper dos isolados bacterianos de carcaças bovinas inconclusivos na identificação bioquímica para Salmonella spp.

\begin{tabular}{ccccccc}
\hline Isolado & Réplica 1 & Escore & Réplica 2 & Escore & Réplica 3 & Escore \\
\hline 22 & Citrobacter freundii & 2,298 & Citrobacter freundii & 2,275 & Citrobacter freundii & 2,191 \\
31 & Citrobacter freundii & 2,174 & Citrobacter freundii & 2,08 & Citrobacter freundii & 2,027 \\
33 & Citrobacter freundii & 2,269 & Citrobacter freundii & 2,268 & Citrobacter freundii & 2,242 \\
62 & Proteus vulgaris & 2,196 & Proteus vulgaris & 2,172 & Proteus vulgaris & 2,05
\end{tabular}

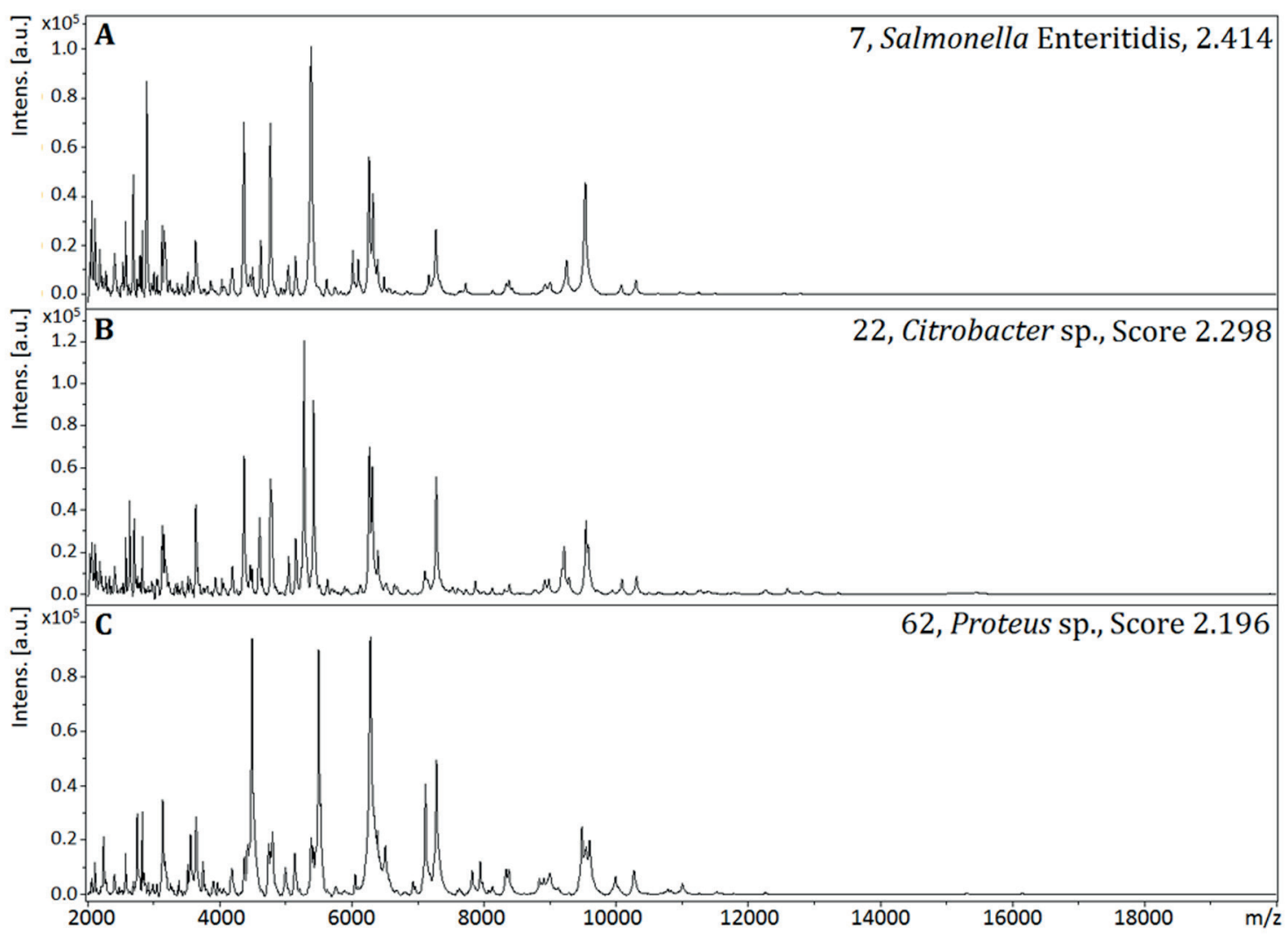

Fig.2. Espectros de massas MALDI-TOF dos isolados 7, 22 e 62 identificados como Salmonella Enteritidis, Citrobacter sp. e Proteus sp. 
Quadro 3. Identificação por MALDI Biotyper dos isolados de carcaças bovinas positivos bioquimicamente para Escherichia coli*

\begin{tabular}{|c|c|c|c|c|c|c|}
\hline Isolado & Réplica 1 & Escore & Réplica 2 & Escore & Réplica 3 & Escore \\
\hline $1355 \mathrm{E}$ & Escherichia coli & 2,539 & Escherichia coli & 2,505 & Escherichia coli & 2,472 \\
\hline 1355L & Escherichia coli & 2,473 & Escherichia coli & 2,468 & Escherichia coli & 2,445 \\
\hline 1358L & Escherichia coli & 2,491 & Escherichia coli & 2,477 & Escherichia coli & 2,449 \\
\hline 247L & Escherichia coli & 2,325 & Escherichia coli & 2,127 & Escherichia coli & 2,126 \\
\hline $320 \mathrm{E}$ & Escherichia coli & 2,5 & Escherichia coli & 2,493 & Escherichia coli & 2,464 \\
\hline $355 R$ & Escherichia coli & 2,376 & Escherichia coli & 2,322 & Escherichia coli & 2,321 \\
\hline $357 \mathrm{E}$ & Escherichia coli & 2,552 & Escherichia coli & 2,442 & Escherichia coli & 2,438 \\
\hline $363 E$ & Escherichia coli & 2,414 & Escherichia coli & 2,39 & Escherichia coli & 2,32 \\
\hline 357R & Escherichia coli & 2,418 & Escherichia coli & 2,39 & Escherichia coli & 2,17 \\
\hline $394 \mathrm{E}$ & Escherichia coli & 2,479 & Escherichia coli & 2,416 & Escherichia coli & 2,241 \\
\hline 395L & Escherichia coli & 2,418 & Escherichia coli & 2,113 & Escherichia coli & 2,082 \\
\hline 398L & Escherichia coli & 2,489 & Escherichia coli & 2,44 & Escherichia coli & 2,425 \\
\hline $399 E$ & Escherichia coli & 2,435 & Escherichia coli & 2,418 & Escherichia coli & 2,399 \\
\hline 399L & Escherichia coli & 2,523 & Escherichia coli & 2,515 & Escherichia coli & 2,508 \\
\hline $401 \mathrm{E} 2$ & Escherichia coli & 2,527 & Escherichia coli & 2,497 & Escherichia coli & 2,449 \\
\hline $402 \mathrm{E} 2$ & Escherichia coli & 2,464 & Escherichia coli & 2,44 & Escherichia coli & 2,412 \\
\hline $453 \mathrm{E}$ & Escherichia coli & 2,369 & Escherichia coli & 2,166 & Escherichia coli & 2,139 \\
\hline $454 \mathrm{E}$ & Escherichia coli & 2,411 & Escherichia coli & 2,391 & Escherichia coli & 2,387 \\
\hline $475 \mathrm{E}$ & Escherichia coli & 2,512 & Escherichia coli & 2,478 & Escherichia coli & 2,458 \\
\hline $476 \mathrm{~L}$ & Escherichia coli & 2,353 & Escherichia coli & 2,313 & Escherichia coli & 2,097 \\
\hline $477 \mathrm{~L}$ & Escherichia coli & 2,373 & Escherichia coli & 2,313 & Escherichia coli & 2,306 \\
\hline $478 \mathrm{E}$ & Escherichia coli & 2,479 & Escherichia coli & 2,434 & Escherichia coli & 2,377 \\
\hline $16 \mathrm{E}$ & Escherichia coli & 2,302 & Escherichia coli & 2,256 & Escherichia coli & 2,247 \\
\hline $17 \mathrm{E}$ & Escherichia coli & 2,444 & Escherichia coli & 2,392 & Escherichia coli & 2,225 \\
\hline $21 \mathrm{E}$ & Escherichia coli & 2,333 & Escherichia coli & 2,282 & Escherichia coli & 2,281 \\
\hline $23 E$ & Escherichia coli & 2,409 & Escherichia coli & 2,336 & Escherichia coli & 2,29 \\
\hline $23 \mathrm{R}$ & Escherichia coli & 2,335 & Escherichia coli & 2,294 & Escherichia coli & 2,195 \\
\hline $6 \mathrm{E}$ & Escherichia coli & 2,448 & Escherichia coli & 2,354 & Escherichia coli & 2,298 \\
\hline $6 \mathrm{~L}$ & Escherichia coli & 2,44 & Escherichia coli & 2,392 & Escherichia coli & 2,387 \\
\hline $7 \mathrm{E}$ & Escherichia coli & 2,397 & Escherichia coli & 2,372 & Escherichia coli & 2,296 \\
\hline
\end{tabular}

* Dos 37 isolados testados por MALDI, sete (354L, 318R, 354E, 356E, 453L, 3L, 7L) apresentaram escore entre 2,0 e 2,299 (dados não monstrados).

cional, demonstraram 95,2\% de consistência na identificação de espécies bacterianas.

A identificação por meio do perfil proteico da bactéria ao invés de diferenciações físicas, metabólicas ou bioquímicas é uma das principais vantagens da espectrometria de massa MALDI-TOF (Hsieh et al. 2008, Angeletti 2016). Neste trabalho, as amostras positivas para Salmonella e $E$. coli identificadas pela microbiologia tradicional foram confirmadas pela análise com MALDI-TOF. Além disso, a técnica MALDI Biotyper possibilitou a identificação dos isolados com perfis bioquímicos não compatíveis com Salmonella spp. ou E. coli, com a vantagem adicional de ser uma técnica bastante mais rápida e possivelmente mais barata.

Ao contrário da identificação por meio de métodos bioquímicos convencionais, que são processos demorados e requerem de 24 a $48 \mathrm{~h}$ após a positividade da cultura, a identificação usando MALDI-TOF pode analisar amostras rapidamente dentro de minutos após a positividade da cultura. De acordo com Panda et al. (2014) o procedimento de extração de proteínas para análise por MALDI-TOF para uma única amostra leva 23 minutos e o tempo de processamento quando feito com várias amostras é reduzido (aproximadamente $3 \mathrm{~h}$ para 82 amostras), facilitando o resultado e aumentando o rendimento. Em outro estudo, realizado por Cherkaoui et al. (2010), ao avaliar 720 amostras em dois métodos de espectrometria de massa MALDI-TOF (Microflex LT e Axima Assurance) frente à identifica- ção convencional bacteriana, os autores concluíram que, se MALDI-TOF tivesse sido aplicado como teste inicial e a identificação bioquímica tivesse sido aplicada apenas quando não havia identificação por MALDI, o laboratório teria economizado cerca de US $\$ 5,00$ por isolado e o tempo para a liberação do resultado teria sido reduzido para aproximadamente 8 horas (Cherkaoui et al. 2010).

Outro aspecto importante é que, apenas uma pequena quantidade do microrganismo é necessária para realizar a análise por MALDI-TOF, sendo possível executar os testes diretamente a partir de colônias isoladas em placas de cultura primárias, enquanto que outros métodos podem requerer outros cultivos (Patel 2013).

A indústria de carnes é um dos principais setores de alimentos no Brasil. A carne bovina é um dos alimentos mais importantes da dieta da população brasileira, além de apresentar um dos maiores potenciais de crescimento e possuir um alto impacto na economia do País. Os resultados deste estudo permitem sugerir a utilização da técnica de MALDI Biotyper em substituição às provas bioquímicas de identificação de patógenos na carne, pois o uso de MALDI-TOF confere maior precisão e rapidez na identificação de eventuais patógenos presentes em carcaças. Isso é de enorme relevância, uma vez que os alimentos de origem animal geralmente possuem uma vida de prateleira curta. Dessa forma, a identificação precisa e rápida de patógenos pode diminuir a perda econômica com alimentos retidos e 
recolhidos, além de implicar em uma melhor qualidade da carne bovina, reduzindo os riscos de infecções humanas de origem alimentícia.

\section{CONCLUSÃO}

A metodologia de espectrometria de massa MALDI-TOF permite a rápida confirmação da identidade de Salmonella spp. e Escherichia coli, isoladas de carcaças bovinas. Essa técnica pode ser utilizada como rotina para identificação de patógenos após isolamento por cultivo de amostras coletadas de abatedouros-frigoríficos.

Agradecimentos.- À Fundação de Apoio ao Desenvolvimento do Ensino, Ciência e Tecnologia do Estado de Mato Grosso do Sul (Fundect) (processo 23/200.479/2014) e à Embrapa Gado de Corte (projetos 03.14.00.047.00.00 e 03.13.10.008.00.00).

\section{REFERÊNCIAS}

APHA 1992. Salmonella, p.371-422. In: Ibid. (Ed.), Compendium of Methods for the Microbiological Examination of Foods. 3rd ed. American Public Health Association, Washington.

Angeletti S. 2016. Matrix assisted laser desorption time of flight mass spectrometry (MALDI-TOF MS) in clinical microbiology. J. Microbiol. (In publication)

Borch E. \& Arinder P. 2002. Bacteriological safety issues in red meat and ready-treat meat products, as well as control measures. Meat Sci. 62(3):381-390.

Brasil 2003. Instrução Normativa no 62 de 26 de agosto de 2003. Métodos analíticos oficiais para análises microbiológicas para controle de produtos de origem animal e água. Ministério da Agricultura, Pecuária e Abastecimento (MAPA), Diário Oficial da União, 18/09/2003, Seção 1, p.14.

Brasil 2016. Animal: Exportação. Ministério da Agricultura, Pecuária e Abastecimento (MAPA). Disponível em <http://www.agricultura.gov. br/animal> Acesso em 6 set. 2016.

Carrique-Mas J.J. \& Davies R.H. 2008. Sampling and bacteriological detection of Salmonella in poultry and poultry premises: a review. Rev. Sci. Tech. OIE 27(3):665-677.

Catellani P., Scapin R.M., Alberghini L., Radu I.L \& Giaccone V. 2014. Levels of microbial contamination of domestic refrigerators in Italy. Food Control 42:257-262.

Cherkaoui A., Hibbs J., Emonet S., Tangomo M., Girard M., Francois P. \& Schrenzel J. 2010. Comparison of two matrix-assisted laser desorption ionization-time of flight mass spectrometry methods with conventional phenotypic identification for routine identification of bacteria to the species level. J. Clin. Microbiol. 48(4):1169-1175.

Clark A., Kaleta E., Arora A. \& Wolk D. 2013. Matrix-assisted laser desorption ionization-time of flight mass spectrometry: a fundamental shift in the routine practice of clinical microbiology. Clin. Microbiol. Rev. 26(3):547-603.

EFSA 2015. The European Union summary report on trends and sources of zoonoses, trends and sources of zoonoses, zoonotic agents and foodborne outbreaks in 2014. European Center for Disease Prevention and Control (ECDC), European Food Safety Authority. EFSA J. 13(12):1-191.

Eigner U., Holfelder M., Oberdorfer K., Betz-Wild U., Bertsch D. \& Fahr A.M. 2009. Performance of a matrix-assisted laser desorption ionizationtime-of-flight mass spectrometry system for the identification of bacterial isolates in the clinical routine laboratory. Clin. Lab. 55(7/8):289-296.

Fagerquist C.K., Zaragoza W.J., Sultan O., Woo N., Quiñones B., Cooley M.B. \& Mandrell R.E. 2014. Top-down proteomic identification of Shiga toxin 2 subtypes from Shiga toxin-producing Escherichia coli by matrix-assisted laser desorption ionization-tandem time of flight mass spectrometry. Appl. Environ. Microbiol. 80(9):2928-2940.

Faron M.L., Buchan B.W., Hyke J., Madisen N., Lillie J.L., Granato P.A., Wilson D.A., Procop G.W., Novak-Weekley S., Marlowe E., Cumpio J., Griego-Full- bright C., Kindig S., Timm K., Young S. \& Ledeboer N.A. 2015. Multicenter evaluation of the Bruker MALDI Biotyper CA system for the identification of clinical aerobic gram-negative bacterial isolates. PLoS One 10(11):e0141350.

FDA 2014. Salmonella, chapter 5. In: Ibid. (Ed.), Bacteriological Analytical Manual. Food and Drug Association, Washington, D.C. Disponível em <http://www.fda.gov/Food/FoodScienceResearch/LaboratoryMethods/ucm2006949.htm> Acesso em 16 ago. 2016.

Filippis F., Storia A., Villani F. \& Ercolini D. 2013. Exploring the sources of bacterial spoilers in beefsteaks by culture-independent high-throughput sequencing. PLoS One 8(7):e70222.

Freiwald A. \& Sauer S. 2009. Phylogenetic classification and identification of bacteria by mass spectrometry. Nature Protocols 4(5):732-742.

Hart P., Wey E., McHugh T.D., Balakrishnan I. \& Belgacem O. 2015. A method for the detection of antibiotic resistance markers in clinical strains of Escherichia coli using MALDI mass spectrometry. J. Microbiol. Meth. 111:1-8.

Havelaar A.H., Brul S., de Jong A., de Jonge R., Zwietering M.H. \& Ter Kuile B.H. 2010. Future challenges to microbial food safety. Int. J. Food Microbiol. 139(1):S79-S94.

Hsieh S.Y., Tseng C.L., Lee Y.S., Kuo A.J., Sun C.F., Lin Y.H. \& Chen J.K. 2008. Highly efficient classification and identification of human pathogenic bacteria by MALDI-TOF MS. Mol. Cell Proteomics 7(2):448-456.

ISO 2002. Microbiology of food and animal feeding stuffs: horizontal method for the detection of Salmonella spp. International Organization for Standardization ISO 6579:2002, Switzerland. 20p.

Lee K.M., Runyon M., Herrman T.J., Phillips R. \& Hsieh J. 2015. Review of Salmonella detection and identification methods: aspects of rapid emergency response and food safety. Food Control 47:264-276.

Lopez-Velasco G., Tomas-Callejas A., Sbodio A.O., Pham X., Wei P., Diribsa D. \& Suslow T.V. 2015. Factors affecting cell population density during enrichment and subsequent molecular detection of Salmonella enterica and Escherichia coli 0157:H7 on lettuce contaminated during field production. Food Control 54:165-175

Marin V.A., Lemos A.A. \& Freitas E.I. 2006. Detecção de patógenos presentes nos alimentos: A falta de padronização e validação de métodos moleculares no Brasil. Rev. Hig. Alim. 20(145):46-50.

Meng J.H., Feng P. \& Doyle M.P. 2001. Pathogenic Escherichia coli, p.331342. In: Downes F.P. \& Ito K. (Eds), Compendium of Methods for the Microbiological Examination of Foods. 4th ed. American Public Health Association, Washington, D.C.

Nagy E., Maier T., Urban E., Terhes G. \& Kostrzewa M. 2009. Species identification of clinical isolates of Bacteroides by matrix-assisted laser-desorption/ionization time-of-flight mass spectrometry. Clin. Microbiol. Infect. 15:796-802.

Panda A., Kurapati S., Samantaray J.C., Srinivasan A. \& Khalil S. 2014. MALDI-TOF mass spectrometry proteomic based identification of clinical bacterial isolates. Indian J. Med. Res. 140:770-777.

Patel R. 2013. Matrix-Assisted laser desorption ionization-time of flight mass spectrometry in clinical microbiology. Clin. Infect. Dis. 57(4):564572.

Rhoades J.R., Duffy G. \& Koutsoumanis K. 2009. Prevalence and concentration of verocytotoxigenic Escherichia coli, Salmonella enterica and Listeria monocytogenes in the beef production chain: a review. Food Microbiol. 26(4):357-376.

Sauer S., Freiwald A., Maier T., Kube M., Reinhardt R., Kostrzewa M. \& Geider K. 2008. Classification and identification of bacteria by mass spectrometry and computational analysis. PLoS One 30(7):e2843.

Settanni L. \& Corsetti A. 2007. The use of multiplex PCR to detect and differentiate food and beverage-associated microorganisms: a review. J. Microbiol. Meth. 69(1):1-22.

Spanu T., De Carolis E., Fiori B., Sanguinetti M., D’Inzeo T., Fadda G. \& Posteraro B. 2011. Evaluation of matrix-assisted laser desorption ionizationtime-of-flight mass spectrometry in comparison to $r p o B$ gene sequencing for species identification of bloodstream infection staphylococcal isolates. Clin. Microbiol. Infect. 17:44-49. 